\title{
Player Adaptive Entertainment Computing (PAEC): Mechanism to Model User Satisfaction by Using Neuro Linguistic Programming (NLP) Techniques
}

\author{
Andrew Chiou, Member, IEEE and Kok Wai Wong, Senior Member, IEEE
}

\begin{abstract}
The concept of Player Adaptive Entertainment Computing (PAEC) is introduced to provide personalized experiences when interacting with the entertainment media. Two of the important areas in PAEC are to create specific targeted strategies to cater for individual user, and to perform personalization. To accomplish this, a suitable mechanism to model user experience is required. This is explored in a case study using Neuro Linguistic Programming (NLP) that models the NLP spatial anchors of experiences of a sample group of users during game play. These NLP anchors are reconstructed during game play of a second group of users allowing them to experience the same level satisfaction as the first sample group.
\end{abstract}

\section{INTRODUCTION}

Entertainment computing covers areas ranging from computer and digital games, mobile content delivery for entertainment, interactive media, entertainment robot, sociology and psychology in entertainment, and virtual/augmented reality for entertainment [1]. Entertainment computing has becoming a significant area in the field of computing. Recently, it can be observed that there is a shift in focus on the design of the entertainment media for individuals, so as to increase perceived value.

In order to increase the competitiveness and profit margin, many interactive media research and development department of related organizations have in turn shifted their focus in developing something that can increase the perceived value of the user. This is similar to the customer relationship management (CRM) used in the business world [2]. One of the important models in CRM is personalization, which is to provide perceived value to customer when interacting with a business. In entertainment computing industry, the objectives of the designer or developer are quite similar to that of the business, which is to increase the perceived value when interacting with the entertainment media.

In this paper, the concept of Player Adaptive Entertainment Computing (PAEC) has been re-examined. The fundamental of the PAEC is to provide personalized

Andrew Chiou is with the School of Computing Sciences, CQUniversity Australia, Rockhampton Campus, Queensland 4702, Australia (e-mail: a.chiou@cqu.edu.au).

Kok Wai Wong is with the School of Information Technology, Murdoch University, South St., Murdoch, Western Australia 6150 (e-mail: k.wong@murdoch.edu.au). experience for each individual when interacting with the entertainment media [3]. The more common area in entertainment computing is digital games. Therefore for such application, PAEC focuses to create specific targeted strategies to cater for individual game player, and also to perform personalization. Personalization in games can be in terms of difficulty levels, game resources, emotion, characters, etc. In game design, the first area can be easily identified and addressed. However, the second area of personalization is somewhat more challenging to attain. This is due to the differences of players in term of their personality, background, culture, skill and learning abilities. The focus of this paper is to use Neuro Linguistic Programming (NLP) as a mechanism to model user's experience when interacting with the media. The outcome of the models will then be able to help contribute to player satisfaction in the overall model of the PAEC.

\section{PAEC CONCEPT}

Perceived value by the users has a lot of power to determine the success of an entertainment media, and thus PAEC is introduced to address this important issue. There are three broad areas of focus in the PAEC: (1) the player; (2) the content; and (3) the entertainment quality. The interaction between the content and player is driven primarily by the value the player perceives. PAEC can thus be modeled as follow:

$$
\text { perceived } \text { value }_{i}=\frac{\text { experience }+ \text { entertainment quality }}{\text { price }}
$$

We can see from equation (1) that the perceived value for player $i$ has several components. The first component, which is experience, refers to the idea that players buy experience and not the particular entertainment product. To the extent, the content of a product enhances the experience and it then increases the player's perceived value. The content that normally enhances experience in entertainment computing includes game resources, platform and the delivery medium. The entertainment quality also increases player's perceived value. Entertainment quality refers to the ways in controlling the mind of the players so that the player feels entertained, which includes inherent interest of events, indirect control techniques, psychological proximity, and 
player modeling. The way in which a media is produced is often as important as the content itself. Price is also a component of the perceived value. Different components of the player's perceived value provide opportunities for enhancement and management of the interactive content with individual players. From equation (1), we can see that the perceived value is defined at the individual level (hence the subscript $i$ ). Therefore, it is important to identify the components of perceived value that are unique to each player or player base.

The most important part of the PAEC is related to the perceived value from the game player. As discussed, the experience and entertainment quality are two very important components in the perceived value. Normally, these two factors can be directly related to the factors such as fun, challenge, entertainment, interest level etc. As individuals are different, personalization becomes the important factor to improve this perceived value. Personalization for PAEC is defined as any set of actions that can tailor the entertainment media experience to a particular user or player.

\section{PlayeR FOCUS}

In [4], personalization can have a direct relationship with the psychological aspect of human needs. It is thus suggesting that when personalization in entertainment computing is placed in the PAEC concept, the perceived value which depends on the important component of experience and entertainment quality could also largely depend on the background, culture, belief, and the gender of the game player. It is thus important to explore ways to provide player adaptation in entertainment computing to improve the perceived value. In [5], an intelligent adaptive game system using neural networks has been used to handle one of the straightforward ways in handling personalization, which is the difficulty level. Other approaches in providing personalization in games are player centered game design and player modeling.

Player centered game design aims to improve the game design and development from an individual perspective. The aim of taking a player centered design approach is to enhance the game play experiences regardless of gender, age or experience [6]. On the other hand, some researchers proposed that the concept of player modeling $[6,15]$. Player modeling should be able to enhance the game play experience and provide customization for individuals [6]. Player modeling involves creating a model of different types of players and grouping them based on their experience from the game. This model is then used to adjust game play experiences to meet the players' preference. The ability to group or label players into appropriate/correct classes is always a difficult problem. Often the player types are too specific to a genre, culture or platform or inversely they are too broad and do not provide enough variation to meet the needs of different players. Normally, in order to force a game player into a group, simple assumptions on demographics, gender and player style are often made. All these could be part of the PAEC concept, however, in order to realize the full potential of such concept, more need to be done.

\section{BACKGROUND: NeURO Linguistic PROGRAMMING}

Neuro linguistic programming (NLP) ${ }^{1}$ is based on the work by Bandler and Grinder [10] on modeling the quality of human experiences through the capture of patterns of the stimulants that trigger these experiences. The concept uses the mechanism of spatial anchoring. Spatial anchoring allows key points in the captured pattern to be reconstructed, thus allowing a second party to be influenced by similar stimulants as experienced by the first party. Later works contributed to the formalization in the codification of NLP spatial anchors [11]. The assumption of the foundation of NLP is that mental representation of a person's experience (e.g. sensation, emotion, personal choices) fundamentally consists of patterns of stimulants and its associated strengths as perceived through the visual $(V)$, auditory $(A)$ and kinesthetic $(K)$ senses.

Fig. 1 illustrates the concept of the mechanics of NLP in the context of this paper. The overall progression is initiated in the passive phase where stimulant of types, $V$, A and/or $K$ can affect a person, Person $A$. The resulting effect as experienced by Person $A$ is an experience, experience $x$ (e.g. happy experience). In this phase, the triggering stimulants can be codified in conjunction with the modeling of experience $x$. The product of this codification and modeling is a catalogue of NLP spatial anchors. For example, a person who encounters a combination of a bright flash (visual), a loud bang (auditory) and sudden cold (kinesthetic) will construe this experience as being fear. Hence, from the following implication for NLP spatial anchors,

$$
\operatorname{Trigger}(V+A+T) \rightarrow \operatorname{subject}_{n} \rightarrow \operatorname{Experience}(x)
$$

we obtain,

$$
\text { Trigger(flash }+ \text { bang }+ \text { cold }) \rightarrow \text { Person } \mathrm{A} \rightarrow \text { Experience }(\text { fear })
$$

In Fig. 1, the active phase allows previous anchors to be reconstructed, thereby influencing a person (other than Person A), Person B, to experience a similar experience, i.e. copy of experience $x$. Therefore, the following implication,

$$
\operatorname{Reconstruction}(V+A+T) \rightarrow \operatorname{subject}_{n+1} \rightarrow \operatorname{Copy}(x)
$$

\footnotetext{
${ }^{1}$ Note that NLP in this context refers to Neuro Linguistic Progamming as opposed to Neural Linguistic Programming used in the discipline of artificial neural networks. These two terminologies are not related and refer to two entirely different subject areas.
} 
we again obtain,

Reconstruction $($ flash + bang + cold $) \rightarrow$ Person $B \rightarrow$ Copy $(f e a r)$

The following sections will focus on the implication of NLP techniques in PAEC personalization.

\section{FormaliZATION OF NLP MECHANISM IN ENTERTAINMENT COMPUTING}

In line with the concept of PAEC, this adaptation of NLP attempts to address the issue of personalization of computer games. This section formalizes and demonstrates how NLP techniques can be used as a mechanism to provide optimal experience as defined in (1). Based on simplified NLP parameters, a player's experience during game play can be formalized as follows.

A single experience, independent of time and duration, can be defined as follows,

$$
\text { experience }=f\left(V_{s t}, A_{s t}, K_{s t}\right)
$$

Where, in the context of this paper, NLP spatial anchors are defined as parameters identifying the different stimulants as encountered by the recipient during game play. These parameters are:

$V_{\text {st }}=$ strength of visual stimulant,

$A_{s t}=$ strength of auditory stimulant,

$K_{s t}=$ strength of kinesthetic stimulant.

The function in (2) is defined as the aggregation of the strengths of the NLP parameters.

$$
f\left(V_{s t}, A_{s t}, K_{s t}\right)=\sum\left(\overrightarrow{z_{1} \cap z_{2} \cap z_{3}}\right)
$$

Where the possible values of $z_{n}$ are the measured strengths of the respective $V_{s t}, A_{s t}$ and $K_{s t}$ parameters.

Note that in (3), the aggregation is a vector of ordered individual strength of the NLP parameters, hence the directed bar. That is, the elements of the summation must be listed exactly in the order that the players experience the $V_{s t}$, $A_{s t}$ or $K_{s t}$ stimulants.

Therefore, in the event that (3) successfully models the optimal strength of a player's experience during game play, the resulting expression will be $\sum\left(\overrightarrow{z_{1} \cap z_{2} \cap z_{3}}\right)$. In relation to the theory of NLP, these are the spatial anchors that allows reconstruction of certain experiences that allow a different player's ability to 're-experience' this.

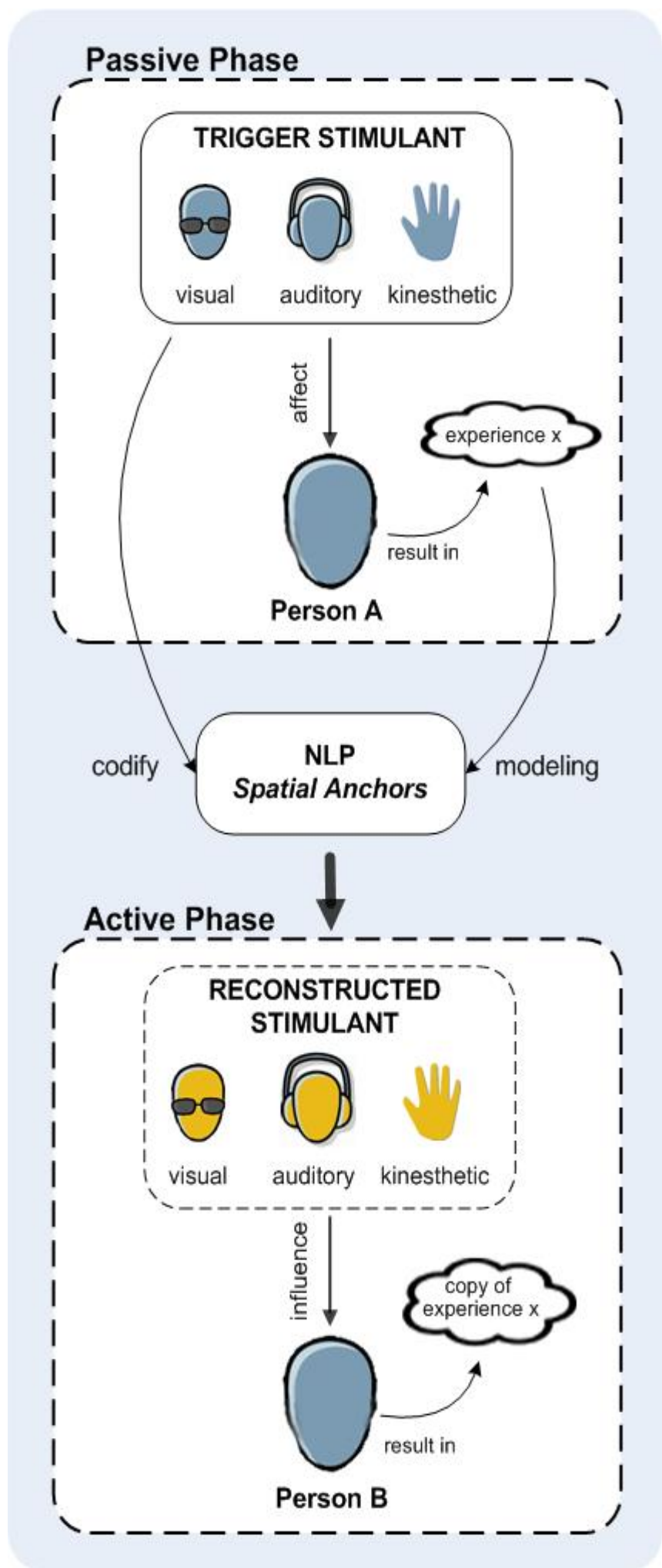

Fig. 1. Progression of NLP mechanism in the codification and reconstruction of NLP spatial anchors. 
However, an experience as encountered by players may be compounded as a series of a collection of singular experiences. By extending (2),

$$
\begin{gathered}
\text { experience }_{\text {complex }}=\text { experience }_{1}+\text { experience }_{2}+ \\
\ldots+\text { experience }_{n}
\end{gathered}
$$

Expanding using (2), we obtain

$$
\begin{aligned}
\text { experience }_{\text {complex }} & =f\left(V_{s t}, A_{s}, K_{s t}\right)_{1}+f\left(V_{s t}, A_{s t}, K_{s t}\right)_{2}+ \\
& \ldots+f\left(V_{s t}, A_{s t}, K_{s t}\right)_{n}
\end{aligned}
$$

Hence, by reconstructing the NLP stimulants against similar factors in game play by using (2) and/or (5), this should ideally reproduce the same level of user experience for subjects from a similar ethnographic (i.e. culture, age group, gender, etc.) background. Henceforth, the following hypothesis is formed:

Hypothesis: In reconstructing (i.e. 'replaying') previously modeled NLP stimulants (spatial anchors) during game play of user $A$, a subsequent user B, should be influenced to experience similar experiences as encountered by user A. [Assumptions are: (a) User A can be an individual or a group; (b) User B is an individual; (c) User A and B are of similar ethnographic background; and (d) the games played are identical.]

The following sections will describe a proposed framework on how NLP spatial anchoring can be used for personalization to support the concept of PAEC.

\section{ROLE OF NLP SPATIAL ANCHORING IN GAMES}

With the advancement of game AI, adaptive game systems could be one of the solutions for producing a player centered game. There are a number of research attempts to use Game AI to manage the adaptive ability of the games [5, 8, 9, 12, 13]. T. Kaukoranta et al. [7] defines adaptation as the ability to react and make appropriate reaction to the change in the interacting environment. By extension, an adaptive game system is defined as a method for creating dynamic heuristic models of player types that adjust themselves over time based on input and measurement from the individual players $[5,6]$. To enable NLP mechanism to co-exist in a game system, modifications has been made to an earlier framework proposed by Charles, D et al [6]. See Fig. 2.

A game system can be any type of digital game that allows a user to interact with its processes (i.e. the gaming functions). To allow adaptation, a monitor function needs to ensure that the game processes are providing optimal experiences to the users. In the proposed framework, the NLP modules are embedded within the monitoring function. As described in the previous section, a baseline is initially established to determine the stereotype of potential users who will be playing the game. This initial stage is the passive phase. The passive phase results in a catalogue of NLP spatial anchors specific to the game. Both functions occur outside the main framework proper and are instantiated once. The catalogue in turn provides all the necessary data to the active phase (residing within the monitor function) to allow it to reconstruct the relevant strengths of the stimulants to trigger personalization within the game systems.

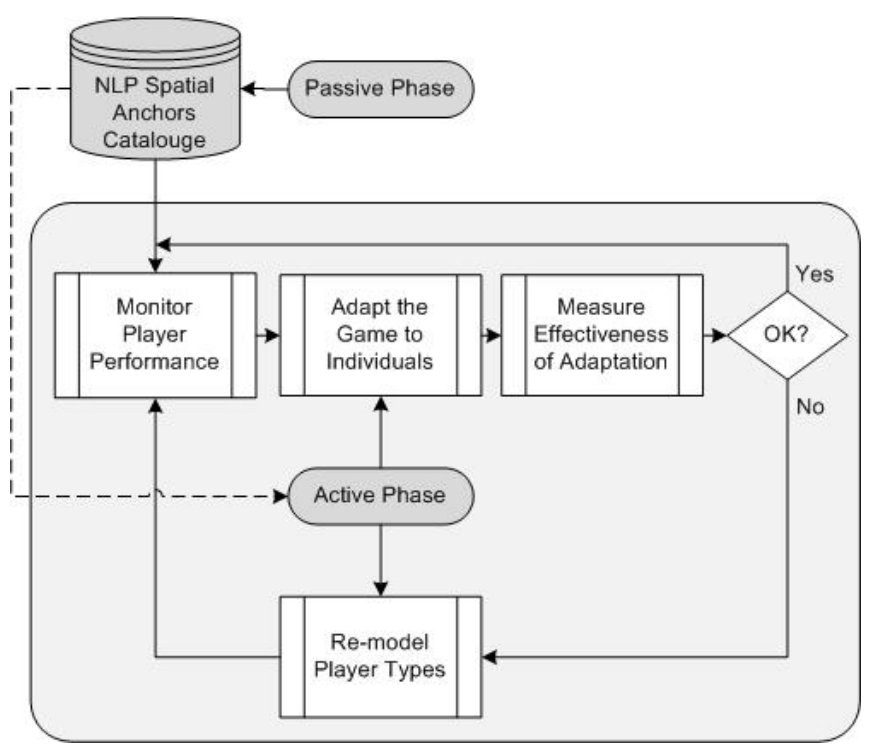

Fig. 2. Modifications made to Charles, D et al. [6] proposed framework to allow NLP mechanism to co-exist in a game systems to provide game adaptation.

\section{PROTOTYPE: XPERT}

The prototype system XperT (Experience Tracker) has been designed and developed to implement the techniques of NLP codification of stimulants and modeling of user experiences during game play. XperT comprises of three distinct modules. These are (1) XperT-PV, (2) X-Factor, and (3) XperT-AV.

The functionality of XperT-PV (Experience Tracker Passive Mode), corresponds to the passive phase depicted in Fig. 1. It captures the strengths of the various NLP visual, auditory and kinesthetic stimulants. The module is implemented as an applet (Fig. 3.), activated concurrently with the game in play. See Fig. 4 and Fig. 5. At specific intervals during game play, users are prompted to enter the intensity of the experience as they have been affected by the three stimulants. Note that the strength of stimulant refers to the level of the strength contributing to the intensity of the experience and does not refer to either the positive or negative sensation or reaction of the player for the respective stimulants.

From an earlier example, a user may experience intense 
fear (i.e. experience $=100$ ). This level of intensity may have been caused by a very bright flash of light (visual $=80$ ), a very loud bang (auditory $=100$ ) and a drastic drop in temperature (kinesthetic $=70$ ). As can be seen none of the above parameters refer to positive sensations or reactions, but to the strengths of each stimulants contributing to the final intensity for experiencing fear.

As a result of the codification and modeling performed by XperT-PV, the collection of distinct NLP spatial anchors is stored in the catalogue, X-Factor (Experience Factor). The source of this catalogue can be either an individual or a group of players for the specific game in question.

XperT-AV (Experience Tracker - Active Mode) corresponds with the active phase Fig. 1. The functionality of this module is to reconstruct the stimulants based on the previously defined spatial anchors during game play to enhance a user's specific experience.

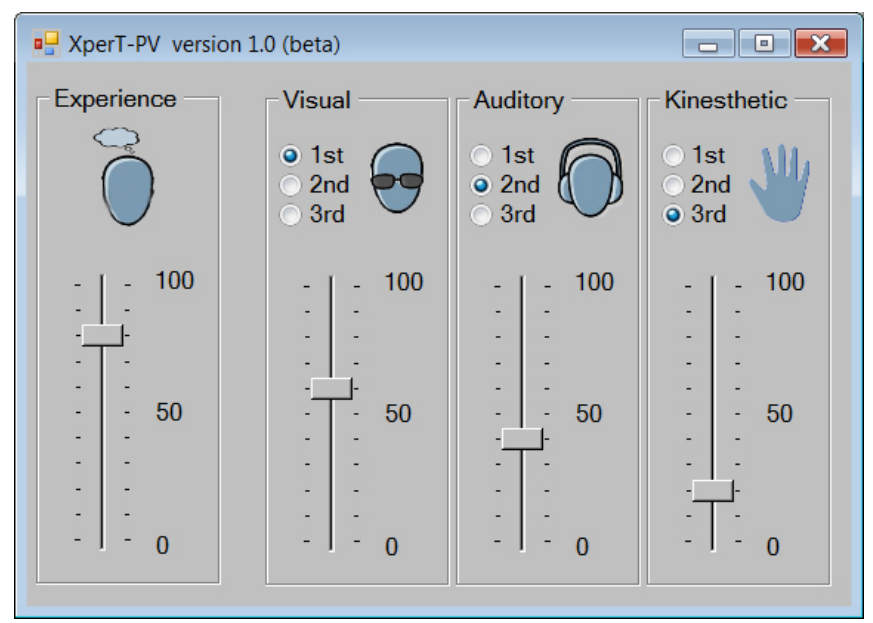

Fig. 3. XperT-PV implemented as an applet to capture the strengths of various NLP $V, A$ and $K$ stimulants.

\section{CASE STUDY}

This section reports a case study to demonstrate the functionality of XperT prototype described in the previous section. A sample $(n=11)$ of chess players from a population of academics were each requested to play a 40 minute game against the computer opponent, Chessmaster: Grandmaster Edition. Each player's ELO [14] playing strength is rated at 1500 or greater. During game play, each subject were prompted every ten minutes (if the games are still in play) to complete the entries on the XperT-PV applet in response to the questions (a) "What intensity of enjoyment are you experiencing during game play?", and (b) "What is the strength of each of the visual, auditory and kinesthetic stimulants affecting this experience (i.e. enjoyment)?". At intervals of ten minutes, coinciding with the above prompts, the game toggled between a Type- $\alpha$ and Type- $\beta$ representation with different emphasis on the $\mathrm{V}, \mathrm{A}$ and $\mathrm{K}$ stimulants. Ten minutes is judged to be the average time between moves where the position of the playing pieces changes, hence the experience of the players and their mental states are interrupted at already naturally interrupted states in order to re-analyse a new board position.

As chess is a game with precise rules and consistent type of game play, it is independent of external factors. This allows the case study to constrain its research focus explicitly to address the hypothesis by effectively isolating the three NLP stimulants from other factors. Here, the case study attempts to determine the spatial anchors for the experience, enjoyment.

Type- $\alpha$ representation (Fig. 4) displays a graphically wellrendered set of standard Staunton-type (i.e. official design for all formal and official chess tournament) chess pieces $(\mathrm{V})$, with audio effects when each pieces are moved (A), and tactile feedback using a Nostromo $n 30$ force-feedback mouse, i.e. users can feel the sensation of holding and moving the chess pieces (K). Type- $\beta$ representation (Fig. 5) displays animated chess pieces of medieval knights (V), with additional audio effects, e.g. sound of swords clashing when a piece captures an opponent (A), and tactile feedback using a Nostromo n30 force-feedback mouse with additional tactile sensation, e.g. players can feel the mouse rumbling during the above sword fights $(\mathrm{K})$.

During the switch between Type- $\alpha$ and Type- $\beta$ representations, the actual state of the game remains constant, i.e., the number of pieces remaining on the chessboard, player's turn, the angle of the chessboard, the playing strength of the chess engine, and position of each remaining pieces remains unaltered. Only the visual, auditory and kinesthetic aspects of the game are toggled between states of Type- $\alpha$ and Type- $\beta$.

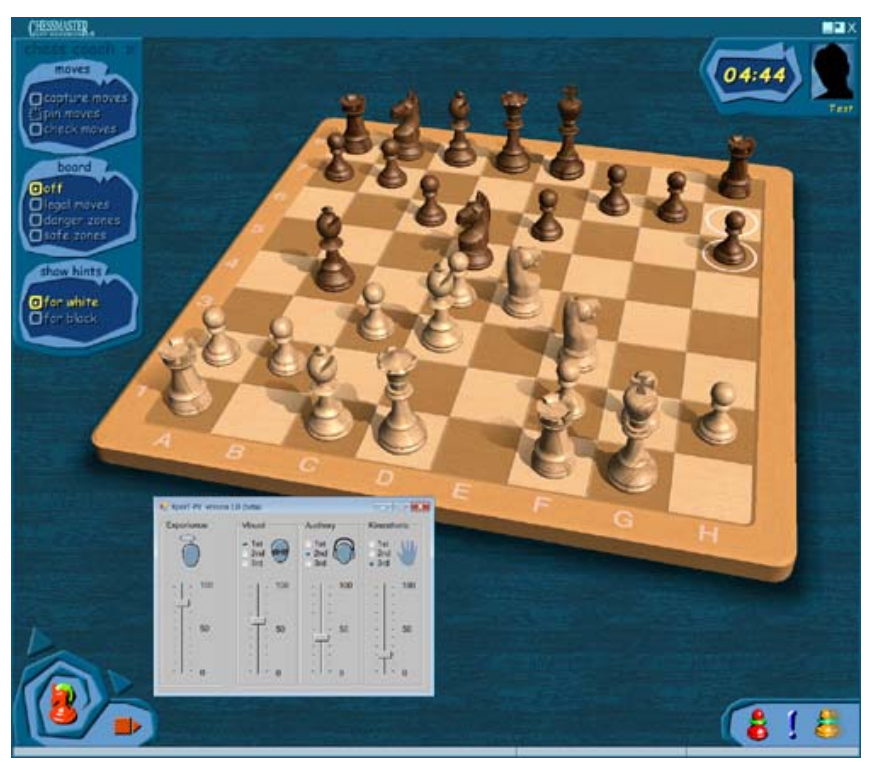

Fig. 4. Type- $\alpha$ representation during game play. 


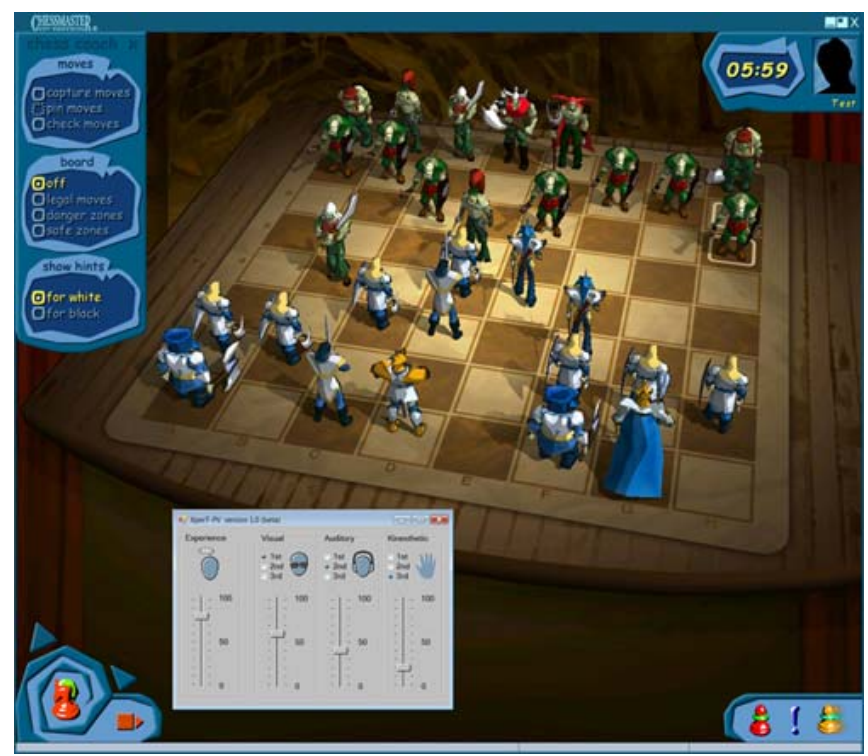

Fig. 5. Type- $\beta$ representation during game play.

The results of XperT-PV is charted in Fig. 6 from Table I. Fig. 6 shows that the average intensity of the subjects experiencing enjoyment is maximized during Type- $\alpha$ representation of the game. It is clearly less so during Type$\beta$ representation. Therefore, the preferred NLP spatial anchors fulfilling the criteria posed in question (a) and (b) are from the Type- $\alpha$ representation.

TABLE I

AVERAGE RESULTS OF THE PASSIVE PHASE

\begin{tabular}{|c|c|c|c|c|}
\hline XperT-PV & Type- $\boldsymbol{\alpha}$ & Type- $\boldsymbol{B}$ & Type- $\boldsymbol{\alpha}$ & Type- $\boldsymbol{B}$ \\
\hline $\boldsymbol{X}$ & 80 & 40 & 80 & 30 \\
\hline $\boldsymbol{V}$-st & 100 & 0 & 100 & 0 \\
\hline $\boldsymbol{A}$-st & 10 & 50 & 10 & 70 \\
\hline $\boldsymbol{K}$-st & 60 & 80 & 60 & 90 \\
\hline
\end{tabular}

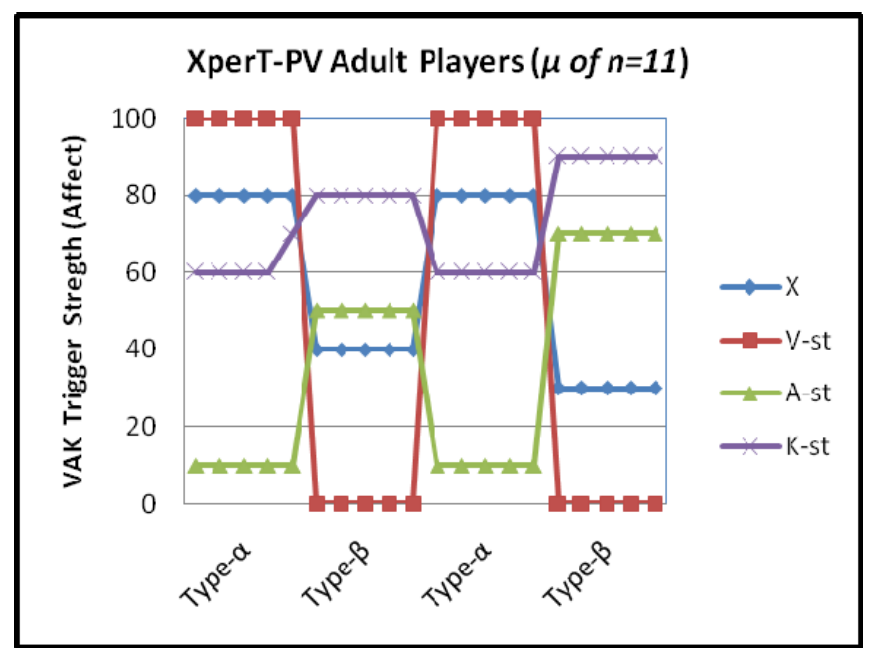

Fig. 6. Intensity of experience as affected by strengths of the various $V, A$ and $K$ stimulants during game play toggling between two different representations of the same game.
A subsequent sample $(n=6)$ of chess players from the same pool (i.e. similar ethnographic background) were further selected. Each subject were requested to play a 40 minute game against Chessmaster with identical settings as to the first group of subjects. To maximize the intensity of each individual subject, only spatial anchors fulfilling the criteria, enjoyment, were constructed. Only Type- $\alpha$ representation was used in the reconstruction. The subjects were prompted only once, to respond to the question (a) and (b), at the end of their games. Note that this phase differs as it no longer attempts to capture the original spatial anchors, but rather to measure the influence of the reconstructed stimulants (i.e. Type- $\alpha$ representation). The resulting measurement of each subject is graphed in Fig. 7 from Table II.

TABLE II

INDIVIDUAL RESULTS OF THE RECONSTRUCTED SPATIAL ANCHORS

\begin{tabular}{|c|c|c|c|c|c|c|}
\hline $\begin{array}{c}\text { XperT- } \\
\text { PV }\end{array}$ & $\begin{array}{c}\text { Player } \\
\mathbf{1}\end{array}$ & $\begin{array}{c}\text { Player } \\
\mathbf{2}\end{array}$ & $\begin{array}{c}\text { Player } \\
\mathbf{3}\end{array}$ & $\begin{array}{c}\text { Player } \\
\mathbf{4}\end{array}$ & $\begin{array}{c}\text { Player } \\
\mathbf{5}\end{array}$ & $\begin{array}{c}\text { Player } \\
\mathbf{6}\end{array}$ \\
\hline $\boldsymbol{X}$ & 80 & 80 & 100 & 90 & 100 & 80 \\
\hline $\boldsymbol{V}$-st & 70 & 90 & 100 & 100 & 80 & 90 \\
\hline A-st & 0 & 20 & 50 & 20 & 10 & 40 \\
\hline K-st & 60 & 100 & 100 & 90 & 100 & 100 \\
\hline
\end{tabular}

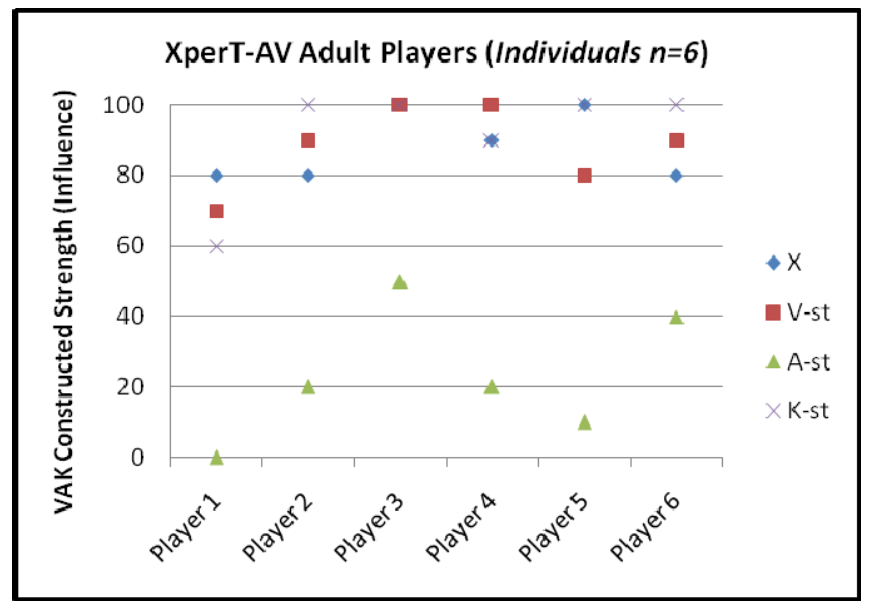

Fig. 7. The influence of reconstructed spatial anchors it has upon players playing against a computer opponent with Type- $\alpha$ representation.

From post-experimental interview with the first group of subjects $(n=11)$, it was determined that adult chess player enjoyed the Type- $\alpha$ representation as the chess pieces 'looked like real pieces', the tactile feedback allows 'the touch-move rule' to be physically realized in a computer game (i.e. minimizes the chance of players touching or moving an unintended piece). However, the subjects found the sound effects affected them minimally, i.e. 'not much difference if there were sound effects or not' and 'chess is a quiet game'. Conversely, the same subjects found Type- $\beta$ representation 'annoying and distracting' as they 'couldn't 
tell the chess pieces apart', the sound effect and tactile feedback 'were inappropriate for a chess game'.

As can be observed from the second sample $(n=6)$ of the reconstructed Type- $\alpha$ representation, the results correlate with the perception of which appropriate stimulants could be duplicated to influence a subject (of similar ethnographic background) to replicate the same intensity of enjoyment while playing the same chess computer game. Therefore, this asserts the hypothesis formalized earlier.

\section{CONCLUSION}

This paper has examined an important success factor that could determine the success of an entertainment computing project. The player is the most important person in the whole development cycle and thus the concept of the PAEC has been investigated. We have also discussed the various developments relating to the PAEC concept. In this paper the Neuro Linguistic Programming (NLP) is used as a mechanism to model user's experience when interacting with the media. This is performed through an XperT prototype. Results from the experiments shows that NLP could be used successfully as a model to increase the level of user satisfaction and thus achieving the goal of PAEC.

\section{ACKNOWLEDGMENTS}

The authors would like to thank the School of Computing Sciences, CQUniversity Australia, for providing the seed grant for the design and development of the XperT prototype.

\section{REFERENCES}

[1] Nakatsu, R. and Hoshino, J. (2002) Entertainment Computing: Technologies and Applications, Kluwer Academic Publishers.
[2] Nykamp, M. (2001) The Customer Differential: The Complete Guide to Implementing Customer Relationship Management, New York, AMACOM.

[3] Wong, K.W. (2008) "Player Adaptive Entertainment Computing," in Proceedings of Computer Games \& Allied Technology 08, April 2008, pp. 32-37.

[4] Oulasvirta, A. and Blom, J. (2007) "Motivations in Personalisation Behaviour," Interacting with Computers, vol. 20, pp. 1-16.

[5] Wong, K.W. (2008) "Adaptive Computer Game System Using Artificial Neural Networks," in Ishikawa, M., Doya, K., Miyamoto, H., Yamakawa, T. (Eds.) Neural Information Processing, Lecture Notes in Computer Science, ISBN: 978-3-540-69154-9, SpringerVerlag, Berlin Heidelberg, pp. 675-682.

[6] Charles, D., McNeill, M., McAlister, M., Black, M., Moore, A., Stringer, K., Kücklich, J. and Kerr, A. (2005) "Player-Centred Game Design: Player Modelling and Adaptive Digital Games," Proceedings of DiGRA 2005 Conference: Changing Views - Worlds in Play (2005).

[7] Kaukoranta T, Smed J, Hakonen H. (2003) "Understanding Pattern Recognition Methods," AI Game Programming Wisdom 2, pp. 579589 .

[8] Ram, A., Ontanon, S., and Mehta, M. (2007) "Artificial Intelligence for Adaptive Computer Games," Proceedings of the Twentieth International FLAIRS Conference on Artificial Intelligence (FLAIRS2007).

[9] Spronck, P. and Herik, J. (2004) "Game Artificial Intelligence that Adapts to the Human Player," ERCIM News, No. 57, April 2004

[10] Bandler, R. and Grinder, J. (1979) Frogs into Princess: Neuro Linguistic Programming. Real People Press.

[11] Dilts, R. and DeLozier, J. (2000) Encyclopedia of Systemic NLP and NLP New Coding. Meta Publications, Capitola, California.

[12] Chiou, A. (2007) "A Game AI Production Shell Framework: Generating AI Opponents for Geomorphic-Isometric Strategy Games via Modelling of Expert Player Intuition", Australian Journal of Intelligent Information Processing Systems, Vol. 9 (4)

[13] Chiou, A, (2007) "What is it Thinking? Game AI Opponent Computer-Human Interaction Using Descriptive Schema and Explanatory Capabilities", Proc. of 2nd International Conference on Digital Interactive Media in Entertainment and Arts (DIMEA) 2007, Perth, Australia.

[14] Elo, A. (1978) The Rating of Chessplayers, Past and Present. Arco.

[15] G. N. Yannakakis, and J. Hallam, (2006) "Towards Capturing and Enhancing Entertainment in Computer Games," in Proceedings of the 4th Hellenic Conference on Artificial Intelligence, Lecture Notes in Artificial Intelligence, vol. 3955, pp. 432-442, Heraklion, Crete, Greece, 18-20 May, 2006. Springer-Verlag. 